PROCEEDINGS OF THE

AMERICAN MATHEMATICAL SOCIETY

Volume 134, Number 1, Pages 187-192

S 0002-9939(05)07931-1

Article electronically published on June 2, 2005

\title{
A SHORT PROOF OF THE SELF-IMPROVING REGULARITY OF QUASIREGULAR MAPPINGS
}

\author{
DANIEL FARACO AND XIAO ZHONG
}

(Communicated by Juha M. Heinonen)

\begin{abstract}
We provide a short proof of a theorem, due to Iwaniec and Martin (1993) and Iwaniec (1992), on the self-improving integrability of quasiregular mappings.
\end{abstract}

\section{INTRODUCTION}

Let $\Omega \subset \mathbf{R}^{n}$ be an open set and let $K$ be a constant greater than one. Then a mapping $f \in W_{\text {loc }}^{1, n}\left(\Omega, \mathbf{R}^{n}\right)$ is said to be $K$-quasiregular if

$$
|D f(x)|^{n} \leq K J(x, f) \quad \text { a.e. } x \in \Omega,
$$

where $|D f(x)|$ is the operator norm of the matrix $D f(x)$, the differential of $f$ at the point $x$, and $J(x, f)$ is the Jacobian of $f$. The theory of quasiregular mappings is a central topic in modern analysis with important connections to a variety of topics such as elliptic partial differential equations, complex dynamics, differential geometry and the calculus of variations [17, [13].

A remarkable feature of quasiregular mappings is the self-improving regularity. In 1957, Bojarski 4 proved that for planar quasiregular mappings, there exists an exponent $p(2, K)>2$ such that quasiregular mappings a priori in $W^{1,2}$ belong to $W^{1, p}$ for every $p<p(2, K)$. In 1973, Gehring 8 extended this result to $n$ dimensional quasiconformal mappings (homeomorphic quasiregular mappings) and proved the celebrated Gehring's lemma. A bit later, Elcrat and Meyers [6] proved that Gehring's ideas can be further exploited to treat quasiregular mappings and partial differential systems; see also [16.

The higher integrability result admits dual version. In two remarkable papers, Iwaniec and Martin [12] (for even dimensions) and Iwaniec [11] (for all dimensions) proved the following theorem.

Theorem 1.1. There exist two numbers $q(n, K)<n<p(n, K)$ such that for every $q, p \in(q(n, K), p(n, K))$, every mapping $f \in W_{\mathrm{loc}}^{1, q}\left(\Omega, \mathbf{R}^{n}\right)$ such that (1.1) holds

Received by the editors May 26, 2004 and, in revised form, August 23, 2004.

2000 Mathematics Subject Classification. Primary 30C65, 35J60.

Key words and phrases. Quasiregular mappings, regularity, Lipschitz approximation.

The first author was supported by the Academy of Finland (Project \# 53292) and by the EU (Project\# HPRN-CT-2002-00282). The second author was supported by the Academy of Finland (Project \# 207288). 
belongs to $W_{\mathrm{loc}}^{1, p}\left(\Omega, \mathbf{R}^{n}\right)$. Moreover, for each $\varphi \in C_{0}^{\infty}(\Omega)$ we have the Caccioppolitype inequality

$$
\|\varphi D f\|_{q} \leq C_{q}(n, K)\|f \otimes \nabla \varphi\|_{q}
$$

where $\otimes$ denotes the tensor product.

The proofs in [11] have been simplified and are nicely presented in [13. The key point is to prove the Caccioppoli inequality for a range of $q$ smaller than $n$. Actually, in the case $q \leq n$, the constant $C_{q}(n, K)$ in the Caccioppoli inequality can be chosen independent of $q$. Then applying the Poincaré inequality, one infers that $|D f|^{q}$ satisfies a weak reverse Hölder's inequality. Then Gehring's lemma can be applied to verify the $L^{q+\delta}$ integrability of $|D f|$ with some $\delta=\delta(n, K)>0$. The exponent will eventually exceed $n$ by iterating the process, and the theorem is proved. The detailed argument is in [13, chapter 14].

It is important to remark that the Caccioppoli inequality yields as well the best available results concerning removable sets for bounded quasiregular mappings. Precisely, based on (1.2), one can prove that sets of $q$-capacity zero, $q>q(n, K)$, are removable; see [13, 17.3].

The proof in [12] and 11] for the Caccioppoli inequality lies very deep. A whole theory about non-linear commutators, non-linear Hodge decompositions and other topics is developed. The even-dimensional situation is almost as complicated.

In this note, we give a short proof of Theorem 1.1. The proof is self-contained and surprisingly elementary. Our approach is inspired by the paper of Lewis [15]. In [15], it is proved that the so-called $A$-harmonic functions also enjoy the selfimproving regularity. Let us recall that each coordinate $f_{i}, i=1,2, \ldots, n$, of a quasiregular mapping $f$ satisfies the equation

$$
\operatorname{div}\left(\left\langle G^{-1} \nabla f_{i}, \nabla f_{i}\right\rangle^{\frac{n-2}{2}} G^{-1} \nabla f_{i}\right)=0,
$$

where $G=J_{f}^{-1} D^{t} f D f$, the distortion tensor of $f$. It is well known that the quasiregularity of $f$ implies that (1.3) is an $A$-harmonic equation. Therefore, the first part of Theorem 1.1 can be alternatively derived from [15. However, we can not argue in this way to prove the Caccioppoli inequality (1.2) for $q<n$, since it is not true, even for $n$-harmonic functions. Hence, to prove (1.2), we need to modify the method in [15] by taking advantage of the fact that the quasiregularity is a condition involving the interplay among all the components of the mapping. Actually, this is the novelty of this note.

The idea of the proof of (1.2) is the following. We construct a Lipschitz continuous function by using a point-wise inequality for the Sobolev functions. More precisely, it is well known that for any mapping $f \in C_{0}^{\infty}\left(\Omega, \mathbf{R}^{n}\right)$,

$$
\int_{\Omega} J(x, f) d x=0 .
$$

An easy approximation argument shows that (1.4) is still true if $f \in W_{0}^{1, n}\left(\Omega, \mathbf{R}^{n}\right)$. Of course, (1.4) is generally not true if we only assume that $f \in W_{0}^{1, q}\left(\Omega, \mathbf{R}^{n}\right)$ for some $q<n$. In order to use the equality (1.4), which is the essential point of the argument, we modify one of the coordinate functions of $f$, say $f_{1}$, by truncating it in terms of the maximal function of $|D f|$ and construct a new function, which is Lipschitz continuous in $\Omega$. Then the equality (1.4) is true for the modified mapping.

The idea of truncating the gradient of the Sobolev functions along the level sets to construct Lipschitz continuous functions was introduced by Acerbi and Fusco 
1], 2. Since then several variations of this idea have been used to treat different problems, in particular after the work of Lewis [15]; see [5, 9], 14, 18]. The method seems to be very effective when dealing with problems involving exponents below the natural ones. This technique will be further exploited to settle the problem of self-improving regularity in the class of mappings of finite distortion [7.

The building block is the following well-known point-wise estimates for the Sobolev functions, whose proofs rely on an argument due to Hedberg [10].

Lemma 1.2 (Point-wise inequalities for the Sobolev functions). Let $u \in W^{1, p}\left(\mathbf{R}^{n}\right)$, $1<q<\infty$, and let $x$ and $y$ be Lebesgue points of $u$ such that $x \in B_{0}=B\left(x_{0}, r\right)$. Then

$$
\begin{gathered}
\left|u(x)-u_{B_{0}}\right| \leq \operatorname{cr} M\left(|\nabla u| \chi_{2 B_{0}}\right)\left(x_{0}\right), \\
|u(x)-u(y)| \leq c|x-y|(M(|\nabla u|)(x)+M(|\nabla u|)(y)),
\end{gathered}
$$

where $c=c(n)>0, \chi_{E}$ is the characteristic function of the set $E, v_{B_{0}}$ is the average of $v$ over $B_{0}=B\left(x_{0}, r\right)$ and $M h$ is the Hardy-Littlewood maximal function of $h$.

Naturally one asks what the exact values of $q(n, K)$ and $p(n, K)$ are. The answer is known in the planar case only. In [3, Astala proved the Gehring-Reich conjecture: the exponents $q(2, K)$ and $p(2, K)$ are $\frac{2 K}{K+1}$ and $\frac{2 K}{K-1}$, respectively. For the higherdimensional cases, there are no good estimates for these thresholds. Unfortunately, our proof does not improve the existing results in this respect.

\section{THE PROOF}

As explained in the Introduction, it is clear that our task is to prove the Caccioppoli inequality with a uniform constant for exponents smaller than $n$. That is, we need to prove that there is $q(n, K)<n$ such that for any $q(n, K)<q \leq n$ if $f \in W_{\text {loc }}^{1, q}\left(\Omega, \mathbf{R}^{n}\right)$ satisfies (1.1), then

$$
\int_{\Omega}|\varphi D f|^{q} d x \leq C(n, K) \int_{\Omega}|f \otimes \nabla \varphi|^{q} d x
$$

for all test-functions $\varphi \in C_{0}^{\infty}(\Omega)$. Clearly we may assume that $\varphi \in C_{0}^{\infty}\left(B_{0}\right)$, $B_{0}=B\left(x_{0}, r\right) \Subset \Omega$, and $\varphi \geq 0$.

We will approximate the first component $f_{1}$ of $f$ suitably. Let $u=f_{1} \varphi$ and extend it to be zero in $\mathbf{R}^{n} \backslash B_{0}$. Then $u \in W^{1, q}\left(\mathbf{R}^{n}\right)$. Denote for $\lambda>0$,

$$
F_{\lambda}=\left\{x \in B\left(x_{0}, r\right): M(g)(x) \leq \lambda \text { and } x \text { is a Lebesgue point of } u\right\},
$$

where $g=|\varphi D f|+|f \otimes \nabla \varphi|$ in $B_{0}$ and $g=0$ in $\mathbf{R}^{n} \backslash B_{0}$. It is easy to show that $u$ is $c \lambda$-Lipschitz continuous on the set $F_{\lambda} \cup\left(\mathbf{R}^{n} \backslash B_{0}\right)$ for $c=c(n) \geq 1$. Indeed, suppose that $x, y \in F_{\lambda}$. Since $|\nabla u| \leq c(n) g$, then it follows from (1.6) that

$$
\begin{aligned}
|u(x)-u(y)| & \leq c|x-y|(M(|\nabla u|)(x)+M(|\nabla u|)(y)) \\
& \leq c|x-y|(M(g)(x)+M(g)(y)) \\
& \leq c \lambda|x-y| .
\end{aligned}
$$

If $x \in F_{\lambda}$ and $y \in \mathbf{R}^{n} \backslash B_{0}$, set $\rho=2 \operatorname{dist}\left(x, \mathbf{R}^{n} \backslash B\left(x_{0}, r\right)\right)$. Since $\mid\{z \in B(x, \rho)$ : $u(z)=0\}|\geq| B(x, \rho) \cap\left(\mathbf{R}^{n} \backslash B_{0}\right)|\geq c(n)| B(x, \rho) \mid$, the Poincaré inequality yields

$$
\left|u_{B(x, \rho)}\right| \leq c(n) \rho|\nabla u|_{B(x, \rho)} \leq c \rho M g(x) \leq c \lambda|x-y| .
$$


Thus by (1.5),

$$
\begin{aligned}
|u(x)-u(y)| & =|u(x)| \leq\left|u(x)-u_{B(x, \rho)}\right|+\left|u_{B(x, \rho)}\right| \\
& \leq c \rho M(|\nabla u|)(x)+c \lambda|x-y| \\
& \leq c \rho M g(x)+c \lambda|x-y| \leq c \lambda|x-y| .
\end{aligned}
$$

If $x, y \in \mathbf{R}^{n} \backslash B_{0}$, then the claim is clear. Since all the other cases follow by symmetry, it follows that $\left.u\right|_{F_{\lambda} \cup\left(\mathbf{R}^{n} \backslash B_{0}\right)}$ is Lipschitz continuous with the constant $c \lambda$. We extend $\left.u\right|_{F_{\lambda} \cup\left(\mathbf{R}^{n} \backslash B_{0}\right)}$ to a Lipschitz continuous function $u_{\lambda}$ in $\mathbf{R}^{n}$ with the same constant by the classical McShane extension theorem. Then we consider the mapping $f_{\lambda}=\left(u_{\lambda}, \varphi f_{2}, \varphi f_{3}, \ldots, \varphi f_{n}\right)$. Since $f \in W_{\mathrm{loc}}^{1, q}\left(\Omega, \mathbf{R}^{n}\right)$, an easy approximation argument shows that if $q \geq n-1$,

$$
\int_{\Omega} J\left(x, f_{\lambda}\right) d x=0
$$

and hence,

$$
\int_{F_{\lambda}} J(x, \varphi f) d x \leq-\int_{\Omega \backslash F_{\lambda}} J\left(x, f_{\lambda}\right) d x .
$$

Now $\left|f_{i} \nabla \varphi\right| \leq C(n)|f \otimes \nabla \varphi|$ and $\left|\nabla\left(\varphi f_{i}\right)\right| \leq c(n) g$. Putting these estimates together with (2.4) and expressing the Jacobian as a wedge product of differential forms, we obtain that

$$
\int_{F_{\lambda}} \varphi^{n} J(x, f) \leq c(n)\left(\lambda \int_{\Omega \backslash F_{\lambda}} g^{n-1}+\int_{F_{\lambda}}|f \otimes \nabla \varphi| g^{n-1}\right) .
$$

This inequality holds for all $\lambda>0$. Then, we multiply it by $\lambda^{-1-\epsilon}$ for some $\epsilon>0$, which will be determined later. We integrate with respect to $\lambda$ over $(0, \infty)$, and finally change the order of integration to obtain that

$$
\begin{aligned}
\int_{\Omega} \varphi^{n} J(x, f) \int_{M g(x)}^{\infty} \lambda^{-1-\epsilon} & d \lambda d x \leq c(n)\left(\int_{\Omega} g^{n-1} \int_{0}^{M g(x)} \lambda^{-\epsilon} d \lambda d x\right. \\
+ & \left.\int_{\Omega}|f \otimes \nabla \varphi| g^{n-1} \int_{M g(x)}^{\infty} \lambda^{-1-\epsilon} d \lambda d x\right) .
\end{aligned}
$$

We use the quasiregularity of $f$, which so far has not been used. It follows that

$$
\begin{aligned}
\int_{\Omega} \varphi^{n}|D f|^{n} M(g)^{-\epsilon} d x & \leq c(n) K \int_{\Omega}|f \otimes \nabla \varphi| g^{n-1} M(g)^{-\epsilon} d x \\
& +\frac{\epsilon c(n) K}{1-\epsilon} \int_{\Omega} g^{n-1} M(g)^{1-\epsilon} d x .
\end{aligned}
$$

From now on, we only use Hölder's inequality and the Hardy-Littlewood maximal theorem to conclude the proof by choosing suitable small $\epsilon$. On the one hand, we have that

$$
\begin{aligned}
\int_{\Omega}(|D f||\varphi|)^{n-\epsilon} d x & \leq\left(\int_{\Omega}|D f|^{n}|\varphi|^{n} M(g)^{-\epsilon} d x\right)^{\frac{n-\epsilon}{n}}\left(\int_{\Omega} M(g)^{n-\epsilon} d x\right)^{\frac{\epsilon}{n}} \\
& \leq c(n)\left(\int_{\Omega}|D f|^{n}|\varphi|^{n} M(g)^{-\epsilon} d x\right)^{\frac{n-\epsilon}{n}}\left(\int_{\Omega} g^{n-\epsilon} d x\right)^{\frac{\epsilon}{n}} .
\end{aligned}
$$

In the second inequality above, we used the Hardy-Littlewood maximal theorem

$$
\int_{\Omega} M(g)^{n-\epsilon} d x \leq c(n) \int_{\Omega} g^{n-\epsilon} d x
$$


where the constant $c(n)>0$ can be chosen so that it does not depend on $\epsilon$ if $0 \leq \epsilon \leq 1$. Now we observe that we may assume

$$
\int_{\Omega} g^{n-\epsilon} d x \leq 2^{n-\epsilon} \int_{\Omega}(|D f||\varphi|)^{n-\epsilon} d x .
$$

Otherwise, the Caccioppoli inequality (2.1) holds with the constant $c(n, K)=1$. Thus it follows from (2.7) that

$$
\int_{\Omega} g^{n-\epsilon} d x \leq c(n) \int_{\Omega}|D f|^{n}|\varphi|^{n} M(g)^{-\epsilon} d x .
$$

On the other hand, we estimate the right-hand side of (2.6) by Hölder's inequality and (2.8). By (2.10) and (2.6),

$$
\begin{aligned}
\int_{\Omega} g^{n-\epsilon} d x & \leq c(n) \int_{\Omega}|D f|^{n}|\varphi|^{n} M(g)^{-\epsilon} d x \\
& \leq c(n) K\left(\int_{\Omega}|f \otimes \nabla \varphi|^{n-\epsilon} d x\right)^{\frac{1}{n-\epsilon}}\left(\int_{\Omega} g^{n-\epsilon} d x\right)^{\frac{n-\epsilon-1}{n-\epsilon}} \\
& +c(n) K \frac{\epsilon}{1-\epsilon} \int_{\Omega} g^{n-\epsilon} d x .
\end{aligned}
$$

Then we conclude the proof by choosing $0<\epsilon<1$ small enough so that

$$
c(n) K \epsilon /(1-\epsilon) \leq 1 / 2 .
$$

\section{REFERENCES}

[1] Acerbi, E.; Fusco, N. An approximation lemma for $W^{1, p}$ functions. Material instabilities in continuum mechanics (Edinburgh, 1985-1986), 1-5, Oxford Sci. Publ., Oxford Univ. Press, New York, 1988. MR0970512 (89m:46060)

[2] Acerbi, E.; Fusco, N. Semicontinuity problems in the calculus of variations. Arch. Rational Mech. Anal. 86 (1984), 125-145. MR0751305 (85m:49021)

[3] Astala, K. Area distortion of quasiconformal mappings. Acta Math. 173 (1994), no. 1, 37-60. MR.1294669 (95m:30028b)

[4] Boyarski B. V. Generalized solutions of a system of differential equations of first order and of elliptic type with discontinuous coefficients. (Russian) Mat. Sb. N.S. 43(85) (1957), 451-503. MR0106324 (21:5058)

[5] Dolzmann, G.; Hungerbühler, N.; Müller, S. Uniqueness and maximal regularity for nonlinear elliptic systems of $n$-Laplace type with measure valued right hand side. J. Reine Angew. Math. 520 (2000), 1-35. MR.1748270 (2001c:35081)

[6] Meyers, N. G.; Elcrat, A. Some results on regularity for solutions of non-linear elliptic systems and quasi-regular functions. Duke Math. J. 42 (1975), 121-136. MR0417568 (54:5618)

[7] Faraco, D.; Koskela, P.; Zhong, X. Mappings of finite distortion: The degree of regularity. Adv. Math. 190 (2005), 300-318. MR2102659 (2005f:30039)

[8] Gehring, F. W. The $L^{p}$-integrability of the partial derivatives of a quasiconformal mapping. Acta Math. 130 (1973), 265-277. MR0402038 (53:5861)

[9] Greco, L. Sharp integrability of nonnegative Jacobians. Rend. Mat. 18 (1998), 585-600. MR 1686812 (2000g:42024)

[10] Hedberg, L. I. On certain convolution inequalities. Proc. Amer. Math. Soc. 36 (1972), 505510. MR0312232 (47:794)

[11] Iwaniec, T. p-harmonic tensors and quasiregular mappings. Ann. of Math. (2) 136 (1992), no. 3, 589-624. MR 1189867 (94d:30034)

[12] Iwaniec, T.; Martin, G. Quasiregular mappings in even dimensions. Acta Math. 170 (1993), no. 1, 29-81. MR1208562 (94m:30046)

[13] Iwaniec, T.; Martin, G. Geometric function theory and non-linear analysis. Oxford Mathematical Monographs. The Clarendon Press, Oxford University Press, New York, 2001. MR:1859913(2003c:30001) 
[14] Koskela, P.; Zhong, X. Hardy's inequality and the size of the boundary. Proc. Amer. Math. Soc. 131 (2003), 1151-1158. MR.1948106 (2004e:26021)

[15] Lewis, J. L. On very weak solutions of certain elliptic systems. Comm. Partial Differential Equations 18 (1993), no. 9-10, 1515-1537. MR.1239922 (94g:35087)

[16] Martio, O. On the integrability of the derivative of a quasiregular mapping. Math. Scand. 35 (1974), 43-48. MR0361064 (50:13510)

[17] Rickman, S. Quasiregular mappings. Springer-Verlag, Berlin, 1993. MR 1238941 (95g:30026)

[18] Zhong, X. On nonhomogeneous quasilinear elliptic equations. Dissertation, University of Jyväskylä, Jyväskylä, 1998. Ann. Acad. Sci. Fenn. Math. Diss. No. 117 (1998), 46 pp. MR.1648847(99j:35075)

Max-Planck-Institute for Mathematics in the Sciences, Inselstr. 22 - 26, 04103 LEIPZIG, Germany

E-mail address: faraco@mis.mpg.de

Department of Mathematics and Statistics, University of Jyväskylä, P.O. Box 35 (MaD), FIN-40014 University OF Jyväskylä, Finland

E-mail address: zhong@maths.jyu.fi 